\title{
Dark and grey electromagnetic electron-cyclotron envelope solitons in an electron-positron magnetoplasma
}

\author{
P.K. SHUK LA A ${ }^{1}$ R. BINGHA M ${ }^{2}$, A. D. R. PHEL P S \\ and L. $\mathrm{S}$ T E N F L O ${ }^{3}$ \\ ${ }^{1}$ Institut für Theoretische Physik IV, Fakultät für Physik und Astronomie, \\ Ruhr-Universität Bochum, D-44780 Bochum, Germany
}

Also at the Department of Physics, Umeå University, SE-90187 Umeå, Sweden; SUPA,

Department of Physics, University of Strathclyde, Glasgow G4 0NG, UK;

Grupo de Lasers e Plasmas, Instituto de Plasmas e Fusão, Instituto Superior Técnico,

Universidade Técnica de Lisboa, 1049-001 Lisbon, Portugal, Max-Planck Institut für extraterrestrische Physik, D-8574l Garching, Germany, and STFC Centre for Fundamental Physics, Rutherford Appleton Laboratory, Chilton, Didcot, Oxfordshire, OX11 0QX, UK (ps@tp4.rub.de)

${ }^{2}$ Scottish Universities Physics Alliance (SUPA), Department of Physics, University of Strathclyde, Glasgow G4 0NG, UK

Also at Space Science and Technology Department, Science \& Technology Facilities Council (STFC), Rutherford Appleton Laboratory, Harwell Science and Innovation Campus, Chilton, Didcot, Oxfordshire, OX11 0QX, UK and STFC Centre for Fundamental Physics, Rutherford Appleton Laboratory, Chilton, Didcot, Oxfordshire OX11 0QX, UK (bob.bingham@stfc.ac.uk; a.d.r.phelps@strath.ac.uk)

${ }^{3}$ Department of Physics, Linköping University, SE-58183 Linköping, Sweden Also at the Department of Physics, Umeå University, SE-90187 Umeå, Sweden (lennart.stenflo@physics.umu.se)

(Received 27 March 2009 and accepted 30 April 2009, first published online 16 June 2009)

\begin{abstract}
We present an investigation of the amplitude modulation of an external magnetic field-aligned right-hand circularly polarized electromagnetic electroncyclotron (EMEC) wave in a strongly magnetized electron-positron plasma. It is shown that the dynamics of the modulated EMEC wave packet is governed by a cubic nonlinear Schrödinger equation. The latter reveals that a modulated wave packet can propagate in the form of either a dark or a grey envelope soliton. This result could have relevance to the transport of electromagnetic wave energy over long distances via envelope solitons in the magnetospheres of pulsars and magnetars.
\end{abstract}

A pair plasma is composed of electrons and positrons, which have the same mass but opposite charge. It exists in the early universe [1-3], in bipolar outflows (jets) in active galactic nuclei $[4,5]$, in the polar regions of neutron stars $[6-13]$, in magnetars $[14,15]$, in the inner regions of the accretion disks surrounding black holes [16], at the centre of our own Galaxy [17], in the Solar atmosphere [18], as well as in plasmas in intense laser fields [19-22], and in tokamaks [23]. Furthermore, due to the production of copious amounts of positrons in some laboratories, one is able to perform experiments on a pair plasma [24-29]. 
In a pair plasma, there are numerous new linear and nonlinear waves [30-41]. The presence of an external magnetic field in a pair plasma greatly affects the dynamics of the pairs via the Lorentz force. Hence, the propagation characteristics of both electrostatic and electromagnetic waves in a pair magnetoplasma are significantly modified [42-57]. In particular, the linear dispersion relations for the nonrelativistic circularly polarized electromagnetic and the non-relativistic elliptically polarized extraordinary electromagnetic waves, which propagate along and across the external magnetic field direction respectively, are the same. Investigations of nonlinear wave-wave interactions in a magnetized pair plasma might play an important role in the description of anti-matter and dark energy of our Universe.

In this letter, we consider the nonlinear propagation of an external magnetic field-aligned negative group dispersion right-hand circularly polarized EMEC wave in a strongly magnetized pair plasma. The external magnetic field is $\hat{\mathbf{z}} B_{0}$, where $\hat{\mathbf{z}}$ is a unit vector along the $z$ axis in a Cartesian coordinate system and $B_{0}$ is the strength of the magnetic field. At equilibrium, we have $n_{\mathrm{e} 0}=n_{\mathrm{p} 0} \equiv n_{0}$, where $n_{0}$ is the unperturbed plasma number density. The wave electric field is $\mathbf{E}_{\perp}=$ $E(\hat{\mathbf{x}}+i \hat{\mathbf{y}}) \exp (-i \omega t+i k z)+$ complex conjugate, where $\hat{\mathbf{x}}$ and $\hat{\mathbf{y}}$ are unit vectors along the $x$ and $y$ axes, respectively. The frequency $\omega$ and the wave number $k$ are related by the cold plasma dispersion relation [44]

$$
\frac{k^{2} c^{2}}{\omega^{2}}=1-\frac{\omega_{\mathrm{p}-}^{2}}{\omega\left(\omega \gamma_{-}-\omega_{\mathrm{c}}\right)}-\frac{\omega_{\mathrm{p}+}^{2}}{\omega\left(\omega \gamma_{+}+\omega_{\mathrm{c}}\right)},
$$

which is valid for $\left|\omega \gamma_{\mp} \mp \omega_{\mathrm{c}}\right| \gg k V_{\mathrm{Te}, \mathrm{Tp}}$. Here $c$ is the speed of light in vacuum, $\omega_{\mathrm{p}}=$ $\left(4 \pi n_{\mp} e^{2} / m\right)^{1 / 2}$ is the electron plasma frequency, $n_{\mp}$ is the sum of the unperturbed and perturbed electron number densities, $e$ is the magnitude of the electron charge, $m$ is the electron rest mass, $\omega_{\mathrm{c}}=e B / m c$ is the electron gyrofrequency, $B$ is the sum of the ambient and perturbed (along $\hat{\mathbf{z}}$ ) magnetic fields, and $V_{\mathrm{Te}}\left(V_{\mathrm{Tp}}\right)$ is the electron (positron) thermal speed. The factor $\gamma_{\mp}=\left(1+\nu_{\mp}^{2}\right)^{1 / 2}$ accounts for the relativistic electron and positron mass increase in the wave field, where $\nu_{\mp}$ is given by $[45-47]$

$$
\nu_{\mp}^{2}=\frac{\Omega_{E}^{2} \gamma_{\mp}^{2}}{\left(\omega \gamma_{\mp} \mp \omega_{c}\right)^{2}},
$$

where $\Omega_{E}=e E / m c$.

Two comments are in order, First, in the non-relativistic limit, we have $\nu_{\mp} \ll 1$ and one can simply approximate $\gamma_{\mp}$ by unity. Then (1a) gives

$$
\frac{k^{2} c^{2}}{\omega^{2}}=1-\frac{2 \omega_{\mathrm{p}}^{2}}{\left(\omega^{2}-\omega_{\mathrm{c}}^{2}\right)}
$$

which for $\omega \approx \omega_{\text {c }}$ and $\omega \ll k c$ yields

$$
\omega \approx \frac{k c \omega_{\mathrm{c}}}{\left(k^{2} c^{2}+2 \omega_{\mathrm{p}}^{2}\right)^{1 / 2}},
$$

where $\omega_{\mathrm{p}}=\omega_{\mathrm{p}-}=\omega_{\mathrm{p}+}$. The wave, given by $(2 \mathrm{~b})$, is referred to as an EMEC wave, which has a negative group dispersion (that is, the rate of change of the group velocity $\partial \omega / \partial k$ with respect to the wave number is negative). 
Second, in the ultra-relativistic limit, we have $\gamma_{\frac{2}{+}}^{2} \gg 1$, and one can approximate $\gamma_{-}$by $\left( \pm \Omega_{E}+\omega_{\mathrm{c}}\right) / \omega$ and $\gamma_{+}$by $\left( \pm \Omega_{E}-\omega_{\mathrm{c}}\right) / \omega$. Here, one obtains from (1a) that

$$
\frac{k^{2} c^{2}}{\omega^{2}}=1 \mp \frac{2 \omega_{\mathrm{p}}^{2}}{\omega \omega_{E}}
$$

which admits the solution

$$
\omega=\frac{2 \omega_{\mathrm{p}}^{2}}{\omega_{E}}\left(1+\frac{k^{2} c^{2} \omega_{E}^{2}}{4 \omega_{\mathrm{p}}^{4}}\right)
$$

with the minus sign in (2c), and a whistler/helicon-like mode [58]

$$
\omega=\frac{k^{2} c^{2}}{2 \omega_{\mathrm{p}}^{2}} \omega_{E}
$$

with the plus sign in $(2 \mathrm{c})$ and with $\omega \omega_{E} \ll 2 \omega_{\mathrm{p}}^{2}$. Both modes, given by (2d) and (2e), have a positive group dispersion.

It is remarkable that the dispersion relation (2c) is independent of the external magnetic field. In addition, (2e) shows that the frequency is proportional to the wave amplitude [45-47]. Furthermore, we observe that in a pair plasma, the whistler/helicon-like mode frequency is half the frequency of ultra-relativistic CPEM waves in a pure electron magnetoplasma $[42,43]$ without positrons.

A finite amplitude weakly relativistic carrier wave $\left(\omega_{0}, k_{0}\right)$ interacting with quasistationary non-resonant fluctuations (composed of the density and magnetic field perturbations) would create an envelope of the wave. The dynamics of a weakly nonrelativistic $\left(\gamma_{\mp} \approx 1\right)$ envelope is governed by a nonlinear Schrödinger equation [30, $52]$

$$
i\left(\frac{\partial}{\partial \tau}+V_{\mathrm{g}} \frac{\partial}{\partial z}\right) E+\frac{V_{\mathrm{g}}^{\prime}}{2} \frac{\partial^{2} E}{\partial z^{2}}-\Delta E=0,
$$

where $\partial E / \partial \tau \ll \omega_{0} E, \omega_{0}=k_{0} c \omega_{\mathrm{c} 0} /\left(k_{0}^{2} c^{2}+2 \omega_{\mathrm{p} 0}^{2}\right), \omega_{\mathrm{c} 0}=e B_{0} / m c$, and $\omega_{\mathrm{p} 0}=$ $\left(4 \pi n_{0} e^{2} / m_{\mathrm{e}}\right)^{1 / 2}$. The group velocity and the group dispersion are $V_{\mathrm{g}}=\partial \omega_{0} / \partial k_{0}=$ $2 c \omega_{\mathrm{c} 0} \omega_{\mathrm{p} 0}^{2} /\left(k_{0}^{2} c^{2}+2 \omega_{\mathrm{p} 0}^{2}\right)^{3 / 2}$ and $V_{\mathrm{g}}^{\prime}=\partial V_{\mathrm{g}} / \partial k_{0}=-6 \omega_{\mathrm{c} 0} \omega_{\mathrm{p} 0}^{2} k_{0} c^{3} /\left(k_{0}^{2} c^{2}+2 \omega_{\mathrm{p} 0}^{2}\right)^{5 / 2}$, respectively. The nonlinear frequency shift $\Delta$ for $\gamma_{\mp} \approx 1$ reads [59]

$$
\Delta=\frac{V_{\mathrm{g}} \omega_{\mathrm{p} 0}^{2}}{k_{0} c^{2}}\left[\frac{2 \omega_{0}^{2} \omega_{\mathrm{c} 0}^{2}}{\left(\omega_{0}^{2}-\omega_{\mathrm{c} 0}^{2}\right)^{2}} \frac{B_{1}}{B_{0}}-\frac{e^{2} \omega_{0}^{2}\left(\omega_{0}^{4}+6 \omega_{0}^{2} \omega_{\mathrm{c} 0}^{2}+\omega_{\mathrm{c} 0}^{4}\right)|E|^{2}}{m^{2} c^{2}\left(\omega_{0}^{2}-\omega_{\mathrm{c} 0}^{2}\right)^{4}}\right],
$$

where $B_{1}$ is the external magnetic field-aligned magnetic field perturbation created by the pressure of the wave envelope due to the inverse Cotton-Mouton effect [60]. The expression for $B_{1}$ is [59]

$$
\frac{B_{1}}{B_{0}}=-\frac{4 \omega_{\mathrm{p} 0}^{2} \omega_{\mathrm{c} 0}^{2}|E|^{2}}{\left(\omega^{2}-\omega_{\mathrm{c} 0}^{2}\right)^{2} B_{0}^{2}} .
$$

We stress that the contribution of the radiation pressure driven density perturbation to the nonlinear frequency shift (4) is insignificant when $\omega_{0} \approx \omega_{\mathrm{c}}$, and can be neglected. One can thus imagine that in a strongly magnetized tenuous plasma with $\omega_{\mathrm{c}} \gg \omega_{\mathrm{p} 0}$, it is reasonable to postulate that the radiation pressure would generate finite magnetic field fluctuations instead of finite plasma number density perturbations. 
Inserting (5) into (3), we have [61]

$$
i\left(\frac{\partial}{\partial \tau}+V_{\mathrm{g}} \frac{\partial}{\partial z}\right) E+P \frac{\partial^{2} E}{\partial z^{2}}+Q|E|^{2} E=0
$$

where we have denoted the group dispersion coefficient $P=V_{\mathrm{g}}^{\prime} / 2$ and the coefficient of the nonlinearity

$$
Q=\frac{V_{\mathrm{g}} \omega_{\mathrm{p} 0}^{2} \omega_{0}^{2} \omega_{\mathrm{c} 0}^{2}}{B_{0}^{2} k_{0} c^{2}\left(\omega_{0}^{2}-\omega_{\mathrm{c} 0}^{2}\right)^{4}}\left(8 \omega_{\mathrm{p} 0}^{2} \omega_{\mathrm{c} 0}^{2}+\omega_{0}^{4}+6 \omega_{0}^{2} \omega_{\mathrm{c} 0}^{2}+\omega_{\mathrm{c} 0}^{4}\right) .
$$

Since the product $P Q$ is negative, (6) depicts that the modulated wave packet is modulationally stable [62]. The stable wavepacket, however, would propagate in the form of a dark/grey envelope soliton [63], the profile of which in a stationary frame has been displayed in various papers [63-67].

Furthermore, we note that the amplitude modulation of the whistler-like mode in our pair magnetoplasma can be readily investigated following the analysis in [53]. Here, however, one has to account for the ponderomotive force driven density fluctuation and relativistic plasma particle mass increase in the wave field. The results in [53] would apply directly, except that for the present case one has to replace $\omega_{\mathrm{p} 0}$ by $\sqrt{2} \omega_{\mathrm{p} 0}$.

To summarize, we have presented an investigation of the amplitude modulation of a weakly relativistic EMEC wave in a strongly magnetized pair plasma, accounting for the radiation pressure driven external magnetic field-aligned quasi-stationary magnetic field perturbation and relativistic electron and positron mass increase in the wave field. It is found that the amplitude modulated wave packet, which has a negative group dispersion, is modulationally stable. A stable nonlinear wave packet can propagate in the form of either a dark or grey envelope soliton. The latter is associated with a magnetic field hole that traps the localized envelope having an insignificant electric field at the center of the envelope. In conclusion, we stress that the wave energy transport could occur via a dark or grey envelope soliton in strongly magnetized pair plasmas, such as those in pulsars and magnetars where the electron gyrofrequency is much higher than the electron plasma frequency.

\section{Acknowledgements}

This work was partially supported by the Deutsche Forschungsgemeinschaft through project SH 21/3-1 of Research Unit 1048, and by an UK EPSRC research grant (at the University of Strathclyde, Glasgow, UK) for carrying out the research work dealing with 'Instabilities in Non-thermal Plasmas'. PKS and RB would like to thank the STFC Centre for Fundamental Physics for support.

\section{References}

[1] Misner, W., Thorne, K. and Wheeler, J. A. 1973 Gravitation. San Francisco: Freeman, p. 763 .

[2] Rees, M. J. 1983 in The Very Early Universe (ed. G. W. Gibbons, S. W. Hawking, and S. Siklas). Cambridge: Cambridge University Press.

[3] Lehnert, B. 2008 Progress in Phys. 1, 16.

[4] Miller, H. R. and Witta, P. 1987 Active Galactic Nuclei. Berlin: Springer, p. 202.

[5] Begelman, M. C., Blandford, R. D. and Rees, M. J. 1984 Rev. Mod. Phys. 56, 255.

[6] Goldreich, P. and Julian, W. H. 1969 Astrophys. J. 157, 869. 
[7] Ginzburg, V. L. 1971 Sov. Phys. Usp. 14, 83.

[8] Sturrock, P. A. 1971 Astrophys. J. 164, 529.

[9] Ruderman, M. A. and Sutherland, P. G. 1975 Astrophys. J. 196, 51.

[10] Michel, F. C. 1982 Rev. Mod. Phys. 54, 1.

[11] Michel, F. C. 1991 Theory of Neutron Star Magnetosphere. Chicago: Chicago University Press.

[12] Gurevich, A. V. and Istomin, Ya. N. 1993 Physics of the Pulsar Magnetosphere. Cambridge: Cambridge University Press.

[13] Asseo, E. 2003 Plasma Phys. Controlled Fusion 45, 853.

[14] Kouveliotou, C., Dieters, S. and Strohmayer T. et al. 1998 Nature (London) 393, 235.

[15] Marklund, M. and Shukla, P. K. 2006 Rev. Mod. Phys. 78, 591.

[16] Rees, M. J. 1971 Nature (London) 229, 312.

[17] Burns, M. L. 1983 Positron-Electron Pairs in Astrophysics (ed. M. L. Burns, A. K. Harding and R. Ramaty). New York: AIP.

[18] Tandberg-Hansen, E. and Emslie, A. G. 1988 The Physics of Solar Flares. Cambridge University Press, p. 124.

[19] Berezhiani, V., Tskhakaya, D. D. and Shukla, P. K. 1992 Phys. Rev. A 46, 6608.

[20] Liang, E. P., Wilks, S. C. and Tabak, M. Phys. Rev. Lett. 1998 81, 4887.

[21] Gahn, C., Tsakiris, G. D. and Witte K. J. et al. 2000 Appl. Phys. Lett. 77, 2662.

[22] Wilks, S. C. Chen, H. and Liang, E., Patel, P., Price, D., Remington, B., Shepherd, R., Tabak, M. and Kruer, W. L. 2005 Astrophys. Space Sci. 298, 347.

[23] Helander, P. and Ward, D. J. 2003 Phys. Rev. Lett. 90, 135004.

[24] Surko, C. M., Leventhal, M. and Passner, P. 1989 Phys. Rev. Lett. 62, 901.

[25] Surko, C. M. and Murphy, T. J. 1990 Phys. Fluids B 2, 1372.

[26] Greaves, R. G., Tinkle, M. D. and Surko, C. M. 1994 Phys. Plasmas 1, 1439.

[27] Greaves, R. G. and Surko, C. M. 1995 Phys. Rev. Lett. 75, 3846.

[28] Gilbert, S. J. Dubin, D. H. E., Greaves, R. G. and Surko, C. M. 2001 Phys. Plasmas 8, 4982.

[29] Greaves, R. G. and Surko, C. M. 2000 Phys. Rev. Lett. 85, 1883.

[30] Shukla, P. K., Rao, N. N., Yu, M. Y. and Tsintsadze, N. L. 1986 Phys. Rep. 138, 1.

[31] Yu, M. Y., Shukla, P. K. and Stenflo, L. 1986 Astrophys. J. Lett. 309, L63.

[32] Stenflo, L., Shukla, P. K. and Yu, M. Y. 1985 Astrophys. Space Sci. 117, 303.

[33] Lominadze, J. G., Stenflo, L., Tsytovich, V. N. and Wilhelmsson, H. 1982 Phys. Scripta 26, 455 .

[34] Gedalin, M. E., Lominadze, J. G., Stenflo, L. and Tsytovich, V. N. 1985 Astrophys. Space Sci. 108, 393.

[35] Iwamoto, N. 1993 Phys. Rev. E 47, 604.

[36] Zank, G. P. and Greaves, R. G. 1995 Phys. Rev. E 51, 6079.

[37] Yu, M. Y., Shukla, P. K. and Stenflo, L. 1986 Astrophys. J. 309, L63.

[38] Stenflo, L., Shukla, P. K. and Yu, M. Y. 1985 Astrophys. Space Sci. 177, 303.

[39] Gangadhara, R. T., Krishan, V. and Shukla, P. K. 1993 Mon. Not. R. Astron. Soc. 262, 151.

[40] Popel, S. I., Vladimirov, S. V. and Shukla, P. K. 1995 Phys. Plasmas 1, 716.

[41] Eliasson, B. and Shukla, P. K. 2006 Phys. Rep. 422, 225.

[42] Kotsarenko, N. Y. 1977 Sov. J. Plasma Phys. 3, 197.

[43] Papuashvili, N. A. Tsikarishvili, E. G. and Tsintsadze, N. L. 1980 Sov. J. Plasma Phys. 6, 331 .

[44] Stenflo, L. 1976 Phys. Scripta 14, 320.

[45] Stenflo, L. and Tsintsadze, N. L. 1979 Astrophys. Space Sci. 64, 513. 
[46] Tsintsadze, N. L., Papuashvili, N. A., Tsikarishvili, E. C. and Stenflo, L. 1980 Phys. Scripta 21, 183.

[47] Stenflo, L., Tomaradze, G. P. and Tsintsadze, N. L. 1983 Beitr. Plasma Phys. 23, 459.

[48] Verheest, F. 1996 Phys. Lett. A 213, 177.

[49] Shukla, P. K. and Stenflo, L. 1996 Pulsars: Problems and Progress (ASP Conference Series, vol. 105) (ed. S. Johnston, M. A. Walker and M. Bailes). San Francisco: ASP, p. 171.

[50] Berezhiani, V. I., Skarka, V. and Mahajan, S. M. 1993 Phys. Rev. E 48, R3252.

[51] Skarka, V., Berezhiani, V. I. and Carlini, G. 1998 Phys. Scripta 57, 456.

[52] Cattert, T., Kourakis, I. and Shukla, P. K. 2005 Phys. Plasmas 12, 012319.

[53] Nishikawa, K., Tsintsadze, N. L. and Watanabe, M. 1980 Sov. J. Plasma Phys. 6, 713.

[54] Shukla, P. K. 2006 J. Plasma Phys. 72, 159.

[55] Stark, C. R., Diver, D. A., de Costa, A. A. and Laing, E. W. $2007 A \& A$ 476, 17.

[56] Shukla, P. K., Brodin, G., Marklund, M. and Stenflo, L. 2008 Phys. Plasmas 15, 082305.

[57] Shukla, P. K. 2009 Plasma Phys. Control. Fusion 51, 024013.

[58] Shukla, P. K. 1978 Nature (London) 274, 874.

[59] Stenflo, L. and Shukla, P. K. 1989 Phys. Rev. B 39, 12941.

[60] Abdullaev, A. Sh. 1988 Sov. J. Plasma Phys. 14, 214.

[61] Shukla, P. K. and Stenflo, L. 1984 Phys. Rev. A 30, 2110.

[62] Hasegawa, A. 1975 Plasma Instabilities and Nonlinear Effects Springer, Berlin.

[63] Hasegawa, A. and Tappert, F. 1973 Appl. Phys. Lett. 23, 171.

[64] Fedele, R. et al. 2002 Phys. Scripta T98, 18.

[65] Fedele, R. 2002 Phys. Scripta 65, 502.

[66] Kourakis, I. and Shukla, P. K. 2003 J. Phys. A. Math. Gen. 36, 11901.

[67] Hasegawa, A. and Matsumoto, M. 2002 Optical Solitons in Fibers. Berlin: Springer. 